\title{
Targeting immunosuppression for cancer therapy
}

\author{
Cristina Ghirelli and Thorsten Hagemann
}

Barts Cancer Institute, John Vane Science Centre, London, United Kingdom.

\begin{abstract}
Failing immunity has been acknowledged for its contribution to cancer development and progression. Recent clinical findings have provided payoffs for significant preclinical evaluation and refinement over the last 20 years, but many questions remain to be answered. In this issue of the JCI, Marabelle et al. describe a novel method for targeting the Tregs that infiltrate tumors, demonstrating that dampening the tumor immunosuppressive environment while activating innate antitumor immunity may be an effective approach to cancer treatment.
\end{abstract}

Immunotherapies have potential for the treatment of cancer, because immunebased therapies act through a mechanism that is distinct from chemotherapy or radiation therapy and because they represent non-cross-resistant treatments, with an entirely different spectrum of toxicities. Both $\mathrm{T}$ and $\mathrm{B}$ cells are capable of recognizing a diverse array of potential tumor antigens through the genetic recombination of their respective receptors, and, more importantly, both $\mathrm{T}$ and $\mathrm{B}$ cells can distinguish small antigenic differences between normal and transformed cells, providing specificity while minimizing toxicity (1).

Several studies have sought to characterize aspects of the immunosuppressive tumor immune microenvironment and the mechanisms that may be responsible (2). There is clinical and preclinical evidence that activation of an antitumor immune response can result in tumor regression and provide clinical benefit, but the natural CTL immunity against tumors often falls short of preventing the development of malignancies. Attempts to maximize the natural response include using antibodies (e.g., anti-CTLA-4-blocking antibodies ipilimumab and ticilimumab) and vaccines (e.g., Provenge) as well as cytokines (e.g., IL-2) (3). However, the clinical response rates to these interventions remain low, and there are currently no clear means to identify either patients who may respond to therapy or identify markers of response in patients that have demonstrated some clinical benefit. Immunotherapy aimed at harnessing endogenous antitumor immunity by modifying immune regulatory

Conflict of interest: The authors have declared that no conflict of interest exists.

Citation for this article: J Clin Invest. 2013; 123(6):2355-2357. doi:10.1172/JCI69999. mechanisms has shown promise in multiple tumor types $(3,4)$. However, in order to unleash the full potential and exquisite specificity of the antitumor immune response and achieve the best clinical responses, the multiple immunosuppressive networks co-opted by tumors need to be defined and collectively overcome (4).

\section{Identifying the regulators}

The adaptive immune system can recognize and eliminate malignant cells; in experimental models of cancer, the adaptive immune system can limit growth of spontaneous and transplanted tumors, and antigen-specific $T$ cells can be detected in human cancers (5). However, the efficacy of this antitumor action is inhibited by the tumor microenvironment. Tolerance to tumor antigen may occur due to antigen persistence, downregulation of MHC, or presence of antigen-specific Tregs; indeed, the prevalence of Tregs in peripheral blood and tumor and expression of programmed death-1 ligand (PDL1) in cancers are independent predictors of poor survival (6). Nonspecific innate tolerance can also be maintained through the production of antiinflammatory and immunosuppressive mediators and downregulation of APC activity (7).

The tumor microenvironment favors immune-suppressive regulators, rather than immune effectors (7). Potential tumor cell-intrinsic mechanisms of immune evasion further include reduced expression of MHC molecules and increased expression of immunosuppressive molecules, e.g., FasL and cytokines, such as IL-10 and TGF- $\beta$. The tumor immune infiltrate is also skewed toward an antiinflammatory and immunosuppressive state, due to the expression of surface molecules that mediate immune suppression like PD-L1 (8).
In addition, tumor-associated macrophages (TAMs), tumor-associated fibroblasts (9), Tregs, and soluble factors produced by suppressor cells all contribute to cancer-induced immune suppression (10). A recent study has also described the contribution of myeloid-derived suppressor cells (MDSCs) to pancreatic cancer progression (11). The accumulation of MDSCs in patients with advanced cancers, including pancreatic cancer, was shown to be closely related to the extent of disease and correlated well with disease stage (12), as did increased infiltration of Tregs, reduced numbers of effector $\mathrm{T}$ cells (e.g., $\mathrm{CD}^{+}$ CTLs), and a bias toward a Th2 response. An increase in Tregs has also been reported in the peripheral blood of patients with cancer with associated impaired response to tumor antigens compared with that to nontumor antigens. TAMs may drive multiple protumor processes, including immunosuppression, angiogenesis, and secretion of direct tumor growth factors (10). The role of other innate immune cell types has not been well characterized.

\section{Targeting immunosuppression}

In this issue of the JCI, Marabelle et al. (13) found that tumor-infiltrating Tregs were enriched for the cell surface markers CTLA-4 and OX40. To target these specifically, the authors injected mouse tumors with anti-CTLA-4 and anti-OX40 antibodies, along with CPG to activate the innate antitumor response. This resulted in a systemic antitumor immune response capable of eradicating disseminated disease. The effect of this immunotherapy was even measurable at distant, therapy-restricted sites like the CNS.

The immunosuppressive markers, targets, and combinational approach described by Marabelle et al. (13) is not entirely novel, as the same group (14) and others $(15,16)$ have already highlighted the importance of combinational immune checkpoint blockade. In clinical trials, findings with CTLA-4 (17) or PD-1 (18-20) antagonists have been encouraging. Patients do respond to the treatment, even if they have advanced disease and are heavily pretreated. However, recent data suggest that Treg infiltration 


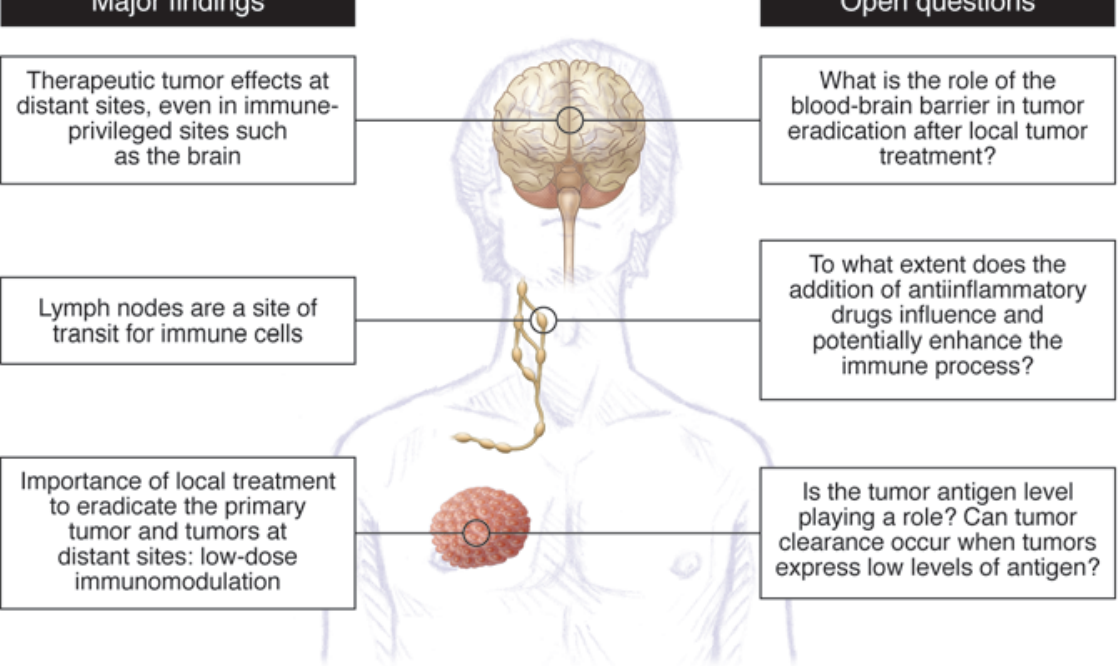

\section{Figure 1}

Marabelle et al. showed how local immunotherapy in mice helps the eradication of tumors at distant sites, even in an immune-privileged site such as the brain. This study opens several questions, and how these findings may translate to human immunotherapy is still a matter of debate.

correlates with better survival (21-24), leaving us puzzled to clinically relate their relevance. Why do only approximately $15 \%$ of patients with advanced melanoma benefit from anti-CTLA-4 treatment? And how can we better screen for those more likely to respond?

\section{Unanswered questions}

The effectiveness of the antibody-mediated immune response is, as outlined above, influenced by several components systemically and within the tumor microenvironment. To what extent does the addition of antiinflammatory drugs influence and potentially enhance the immune response? $\mathrm{F} c \gamma \mathrm{R}$ is expressed on a variety of effector cells, such as macrophages, neutrophils, mast cells, and NK cells, and complement factors are present in the tumor microenvironment, so the interaction of the Fc part of the therapeutic antibody bound to its tumor antigen will initiate an inflammatory response of some kind. This response is crucial to orchestrate the right influx of leukocytes, resulting in lysis of the target cells (antibody-dependent cell cytotoxicity and complement-dependent cytotoxicity). However, it is also clear that cancer-related inflammatory processes in the microenvironment of the tumor mediated by binding of endogenous antibodies can also orchestrate the protumor function of myelomonocytic cells (25). The possibility that these tumor-fostering mechanisms initiated by the therapeutic antibodies can also play a role in tumor treatment and potentially dampen the overall response cannot be excluded.

Perhaps the reason that Marabelle et al. (13) see such a strong systemic effect of their approach is that they guide the immune response away from the immunosuppressive microenvironment of the primary tumor and toward distant sites in which immunosuppression has not yet been established. Do the observations hold true in the absence of a strong antigen? What are the team players involved locally in generating this significant systemic effect? Marabelle et al. certainly observed an effect on lung metastasis from 4T1 tumors; however, will the proposed approach provide an effect on the primary tumor if the metastatic lesion(s) is treated instead? This would be far more feasible for patients in many clinical settings.

As our understanding of the potential of immunotherapy expands, so does the list of research questions that will need to be answered before this approach can be translated for effective clinical use (Figure 1). How long would we need to treat patients with immune modulatory therapies? What is the best combination of approaches? And last, we need a clearly defined clinical readout for therapeutic response. Our understanding of the evolution of immune escape is still incomplete, and additional work must be done to identify those patients who will benefit most from immunotherapy and to develop novel strategies.

\section{Acknowledgments}

Cristina Ghirelli is supported by Pancreatic Cancer Research Fund (PCRF), and Thorsten Hagemann is supported by Cancer Research UK, PCRF.

Address correspondence to: Thorsten Hagemann, Barts Cancer Institute, John Vane Science Centre, Charterhouse Square, 3rd Floor, London, EC1M 6BQ, United Kingdom. Phone: 442078825795; Fax: 442078826110; E-mail: t.hagemann@qmul.ac.uk.

1. Alderton GK, Bordon Y. Tumour immunotherapy--leukocytes take up the fight. Nat Rev Immunol. 2012;12(4):237.

2. Vanneman M, Dranoff G. Combining immunotherapy and targeted therapies in cancer treatment. Nat Rev Cancer. 2012;12(4):237-251.

3. Pardoll DM. Immunology beats cancer: a blueprint for successful translation. Nat Immunol. 2012;13(12):1129-1132.

4. Pardoll DM. The blockade of immune checkpoints in cancer immunotherapy. Nat Rev Cancer. 2012;12(4):252-264.

5. Altman JD, et al. Phenotypic analysis of antigen-specific T lymphocytes. Science. 1996;274(5284):94-96.

6. Hiraoka N, Onozato K, Kosuge T, Hirohashi S. Prevalence of FOXP3 + regulatory T cells increases during the progression of pancreatic ductal adenocarcinoma and its premalignant lesions. Clin Cancer Res. 2006;12(18):5423-5434.

7. Mantovani A, Allavena P, Sica A, Balkwill F. Cancer-related inflammation. Nature. 2008; 454(7203):436-444.

8. Beatty GL, et al. CD40 agonists alter tumor stroma and show efficacy against pancreatic carcinoma in mice and humans. Science. 2011; 331(6024):1612-1616.

9. Kraman M, et al. Suppression of antitumor immunity by stromal cells expressing fibroblast activation protein-alpha. Science. 2010;330(6005):827-830.

10. Balkwill FR, Capasso M, Hagemann T. The tumor microenvironment at a glance. J Cell Sci. 2012; 125(pt 23):5591-5596.

11. Bayne LJ, et al. Tumor-derived granulocyte-macrophage colony-stimulating factor regulates myeloid inflammation and $\mathrm{T}$ cell immunity in pancreatic cancer. Cancer Cell. 2012;21(6):822-835.

12. Diaz-Montero CM, Salem ML, Nishimura MI, Garrett-Mayer E, Cole DJ, Montero AJ. Increased circulating myeloid-derived suppressor cells correlate with clinical cancer stage, metastatic tumor burden, and doxorubicin-cyclophosphamide chemotherapy. Cancer Immunol Immunother. 2009;58(1):49-59.

13. Marabelle A, et al. Depleting tumor-specific Tregs at a single site eradicates disseminated tumors. J Clin Invest. 2013;123(6):2447-2463.

14. Houot R, Levy R. T-cell modulation combined with intratumoral $\mathrm{CpG}$ cures lymphoma in a mouse model without the need for chemotherapy. Blood. 2009;113(15):3546-3552.

15. Curran MA, Montalvo W, Yagita H, Allison JP. PD-1 and CTLA-4 combination blockade expands infiltrating $\mathrm{T}$ cells and reduces regulatory $\mathrm{T}$ and myeloid cells within B16 melanoma tumors. Proc Natl Acad Sci U S A. 2010;107(9):4275-4280.

16. Takeda $\mathrm{K}$, et al. Combination therapy of established tumors by antibodies targeting immune activating and suppressing molecules. J Immunol. 2010;184(10):5493-5501.

17. Hodi FS, et al. Improved survival with ipilimumab in patients with metastatic melanoma. $N$ Engl J Med. 2010;363(8):711-723. 
18. Nomi T, et al. Clinical significance and therapeutic potential of the programmed death-1 ligand/programmed death-1 pathway in human pancreatic cancer. Clin Cancer Res. 2007;13(7):2151-2157.

19. Brahmer JR, et al. Safety and activity of anti-PD-L1 antibody in patients with advanced cancer. $N$ EnglJ Med. 2012;366(26):2455-2465.

20. Topalian SL, et al. Safety, activity, and immune correlates of anti-PD-1 antibody in cancer. $N$ Engl JMed. 2012;366(26):2443-2454.
21. West NR, et al. Tumour-infiltrating FOXP3(+) lymphocytes are associated with cytotoxic immune responses and good clinical outcome in oestrogen receptor-negative breast cancer. $\mathrm{Br} J$ Cancer. 2013;108(1):155-162

22. Yoon HH, Orrock JM, Foster NR, Sargent DJ, Smyrk TC, Sinicrope FA. Prognostic impact of FoxP3+ regulatory $\mathrm{T}$ cells in relation to $\mathrm{CD} 8+\mathrm{T}$ lymphocyte density in human colon carcinomas. PLoS One. 2012;7(8):e42274.
23. Salama $\mathrm{P}$, et al. Tumor-infiltrating FOXP3+ T regulatory cells show strong prognostic significance in colorectal cancer. J Clin Oncol. 2009;27(2):186-192.

24. Lee AM, et al. Number of CD4+ cells and location of forkhead box protein $\mathrm{P} 3$-positive cells in diagnostic follicular lymphoma tissue microarrays correlates with outcome. JClin Oncol. 2006;24(31):5052-5059.

25. Andreu $\mathrm{P}$, et al. FcRgamma activation regulates inflammation-associated squamous carcinogenesis. Cancer Cell. 2010;17(2):121-134.

\title{
Human C3 glomerulopathy provides unique insights into complement factor $\mathrm{H}$-related protein function
}

\author{
V. Michael Holers \\ Departments of Medicine and Immunology, Division of Rheumatology, University of Colorado School of Medicine, Aurora, Colorado, USA.
}

The study in this issue of the JCI by Tortajada et al. demonstrates that a duplication within the gene complement factor $\mathrm{H}$-related 1 (CFHR1; encoding FHR1) leads to the production of an aberrant larger form of the protein. Elegant in vitro studies of the mutant and normal variants demonstrate an unexpected mechanism of action of FHR1, wherein homodimeration and hetero-oligomerization with FHR2 and FHR5 generates more avid molecules that very effectively compete with $\mathrm{FH}$ binding to surfaces and impair its ability to regulate local complement activation. As variants of FHRs are linked to many human inflammatory and autoimmune diseases, these and other recently published structure/function studies of these proteins provide key insights into their complement regulatory activities and likely roles in disease.

\section{Introduction}

Major advances have recently been made in our understanding of the biological and pathophysiological roles of the complement system. Genetic association studies, deep sequencing efforts, clinical association findings, results from animal models, and markedly positive results in therapeutic trials in an increasing number of human diseases have refocused attention on the important pathogenic role of inappropriate complement activation in the broad scope of human diseases $(1,2)$. Complement inhibitors have been successfully developed for therapeutic use in two human genetic deficiency states, hereditary angioedema (HAE; ref. 3) and paroxysmal nocturnal hemoglobinuria (PNH; ref. 4), as well as the rare condition designated atypical hemolytic uremic syndrome (aHUS; ref. 5).

Conflict of interest: $V$. Michael Holers has received consulting income and research support in the last year from Alexion Pharmaceutics.

Citation for this article: J Clin Invest. 2013; 123(6):2357-2360. doi:10.1172/JCI69684
Positive initial clinical trial results have been reported in many additional conditions. Notably, major efforts are underway to understand why common polymorphisms and rare variants of complement pathway genes whose products primarily promote activation of the alternative pathway are associated with human age-related macular degeneration (AMD; ref. 6).

The complement system is an evolutionarily ancient member of the innate immune system that is involved in many inflammatory and autoimmune diseases, with functions ranging from modulation of adaptive immunity to generation of potent injurious effector functions when endogenous control mechanisms fail to restrain its activities during tissue injury (7). Complement system components are in general well understood with regard to their structure/function characteristics. The system consists of more than 40 proteins that either function during activation through the three initiation pathways and the amplification loop or act as recognition molecules, receptors, negative regulatory proteins, or stabilizing/ activating factors (7). There are, however, a number of fundamental unanswered questions with regard to certain components of the system, and high on the list are the functional roles of complement factor $\mathrm{H}-$ related proteins (FHRs).

What are FHRs? As Tortajada et al. note in this issue (8), these proteins have been known for many years to be part of a structurally related family including the larger factor $\mathrm{H}$ protein (FH; encoded by $\mathrm{CFH}$ ). FHRs are encoded by a series of genes adjacent to $C F H$ on human chromosome 1 and also contain short consensus repeat (SCR) domains with homology to subregions of FH $(9,10)$. As genetic variants characterized by the absence of CFHR 1 and CFHR3 $\left(\Delta_{\text {CFHR3-CFHR } 1}\right)$, or of CFHR 1 and CFHR 4 $\left(\Delta_{\text {CFHRI-CFHR }}\right)$, are relatively common in the human population, the proteins encoded by these genes do not appear to be required for human development or immune competence under normal conditions. Nevertheless, an increasing number of protective or risk associations of deletions or variants of these genes have been reported with human diseases $(9,10)$. Among these are AMD, for which $\Delta_{\text {CFHR3 } 3 \text { CFHRI }}$ is a highly penetrant protective factor (11), and aHUS,

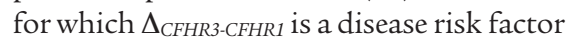
and is associated with autoantibodies that interfere with FH regulatory function (12).

\section{Duplication of sequences within CFHR1 is linked to $\mathrm{C} 3$ glomerulopathy}

Many of the recent advances in the complement field have been driven by study of rare 